\section{$\underset{\substack{\text { hommes } \\ \text { \& migrations }}}{ }$}

\section{Hommes \& migrations}

Revue française de référence sur les dynamiques

migratoires

$1309 \mid 2015$

Le $3 e$ âge des migrants

\title{
Le Prix littéraire de la Porte \#6
}

Le jury et la sélection 2015

\section{Élisabeth Lesne}

\section{OpenEdition \\ 1 Journals}

\section{Édition électronique}

URL : http://journals.openedition.org/hommesmigrations/3126

DOI : 10.4000/hommesmigrations.3126

ISSN : 2262-3353

\section{Éditeur}

Musée national de l'histoire de l'immigration

\section{Édition imprimée}

Date de publication : 1 janvier 2015

Pagination : 202-205

ISBN : 978-2-919040-30-8

ISSN : 1142-852X

\section{Référence électronique}

Élisabeth Lesne, «Le Prix littéraire de la Porte \#6 », Hommes \& migrations [En ligne], 1309 | 2015, mis en ligne le 31 août 2015, consulté le 24 septembre 2020. URL : http://journals.openedition.org/ hommesmigrations/3126; DOI : https://doi.org/10.4000/hommesmigrations.3126 


\title{
LITTÉRATURE
}

\section{LE PRIX LITTÉRAIRE DE LA PORTE DORÉE \#6 LE JURY ET LA SÉLECTION 2015}

\author{
ELISABETH LESNE
}

Après Julien Delmaire, lauréat 2014 pour Georgia (Grasset), découvrez qui sera notre prochain lauréat lors de la fête de remise, le 3 juin à 19 h 00, au Musée national de l'histoire de l'immigration.

\section{Nathacha Appanah \\ En attendant demain \\ Gallimard}

On l'apprend dès la première page : un drame a ravagé la vie d'Adam et Anita, il y a "quatre ans, cing mois et treize jours". Cette menace plane sur eux comme une malédiction quand on les découvre quelque vingt ans plus tôt un soir de réveillon, étrangers tous deux aux codes parisiens. Adam est bordelais, étudiant en architecture; Anita, mauricienne, en fac de lettres. Leur amour leur donne des ailes : ils rêvent de devenir peintre et écrivain, d'“incarner un couple d'artistes, mystérieux, talentueux et amoureux". Après la naissance de leur fille, ils s'installent dans le Sud-Ouest. Lui crée son agence, elle devient "correspondante" dans la presse locale. Pas si mal, mais qu'ont-ils fait de leurs rêves ? L'arrivée d'Adèle, Mauricienne elle aussi, va opérer comme une lame de fond. Apportant sérénité dans la maison et inspiration créatrice au couple. Quelques moments de bonheur volés, juste avant la chute. Un thriller psychologique subtil sur la folie d'un couple ordinaire, fût-il "mixte".

\section{Gaston-Paul Effa \\ Rendez-vous avec l'heure qui blesse \\ "Continents noirs", Gallimard}

Dans ce roman à la première personne, GastonPaul Effa donne sa voix au Martiniquais Raphaël Élizé, le premier maire noir de France métropolitaine. En 1919, celui-ci s'installe comme vétérinaire à Sablé-sur-Sarthe. Dix ans plus tard, à force d'empathie et de travail, ce descendant d'esclave réussit à gagner la confiance de la population rurale et à se faire élire maire, jusqu'à ce que le régime de Vichy le destitue. Homme de grande culture, membre de la SFIO et résistant, Élizé est déporté à Buchenwald. "Tout aura commencé cet automne 1943, où la Gestapo me cueillit chez moi par une nuit enveloppée de brume", lit-on sur la première page. Procédant comme par fondus enchaînés, passant sans cesse du quotidien du prisonnier, où Élizé est renvoyé à sa condition de "nègre" ("j'étais moins qu'un juif, si cela est pensable"), aux souvenirs de sa vie de notable, Gaston-Paul Effa n'écrit pas une biographie, mais un texte à portée philosophique et politique . 


\section{Gauz}

\section{Debout-Payé}

\section{Le Nouvel Attila}

À quoi pensent les vigiles des grandes enseignes qui sont payés, comme le dit le titre dans un joli raccourci, pour rester debout et "répéter cet ennuyeux exploit de l'ennui" ? Après la lecture de Debout-Payé, premier "roman" toujours drôle, percutant et politique de l'Ivoirien Gauz, on considère d'un autre œil ces hommes de l'ombre à la peau sombre qui observent nos faits et gestes. Mi-roman, mi-essai de sociologie, Debout-Payé raconte le parcours de plusieurs générations de vigiles et réunit rappels historiques, notes en vrac et sketchs souvent hilarants sur les clients du Camaïeu de Bastille ou du Séphora des ChampsÉlysées. Efficace pour se moquer de la folie consumériste et de la politique de l'immigration des années 1960 à l'après-11 septembre. Une langue pleine d'énergie émaillée de "nouchi", parler populaire d'Abidjan. Une verve iconoclaste qui se joue des clichés.

\section{Max Lobe}

La Trinité bantoue

Zoé

Avec bravoure et humour, Mwana raconte ses tribulations en Helvétie au moment où tout se met à dérailler dans sa vie : il se fait licencier de chez Nkamba African Beauty et sa mère restée au Bantouland est atteinte d'un cancer. En pleine campagne xénophobe de chasse aux moutons noirs dans un pays au taux de chômage le plus bas d'Europe, ce super-diplômé ne décroche qu'un stage dans une ONG pour la promotion de 


\section{LITTÉRATURE}

la diversité. Son salaire de misère l'oblige à faire appel aux Colis du cœur, un comble alors que son compagnon, Ruedi, un gosse de riche helvète pure souche, refuse de demander de l'aide à sa famille. Rien à voir avec la solidarité qui règne au Bantouland. Ce choc des cultures est raconté dans une langue qui s'éloigne joyeusement du "gros-gros français" et puise dans les proverbes africains: "La vie chez les Blancs, c'est caillou" ? Oui mais "la hyène qui passe son temps à hurler n'aura jamais sa proie". Un récit plein de grâce. Bantoue, peut-être?

\section{Sylvain Prudhomme}

\section{Les Grands}

L’Arbalète/Gallimard

"I muri." C'est par ces mots que commence le roman. "Elle est morte", elle, c'est Dulce, la chanteuse du Super Mama Djombo, que "tout un peuple appelait encore aujourd'hui par son prénom, comme une amie, une sour". Elle s'est mariée avec Gomes, l'homme fort actuel de la Guinée-Bissau, mais le veuf de Dulce, le vrai, c'est Couto, le guitariste du groupe. Baladant ses souvenirs dans l'atmosphère fiévreuse d'un énième coup d'État, Couto déambule, ouvert aux rencontres. Peu à peu la nouvelle enflamme la ville, comme le groupe enflammait les scènes du monde entier au milieu des années 1970. "Une success-story" qui a accompagné I'histoire du pays, de son indépendance aux soubresauts d'un pouvoir corrompu. Bien que la plupart des anciens vivent loin, "Frères dispersés. Galériens des grandes villes (...). Forçats d'une Europe qui voulait bien d'eux, mais comme soutiers, le contraire des rois qu'ils avaient rêvés d'être", un concert rendra hommage à Dulce le soir même. Ce roman de deuil est un hymne à la vie, à l'amour, à l'Afrique, sans une once d'exotisme de pacotille.

\section{Andrea Salajova}

\section{Eastern}

Gallimard

En 2005, dix ans après être parti vivre à Paris, Martin, danseur et chorégraphe, revient en Slovaquie fêter le dernier anniversaire de son grand-père. À Michalovce, petite ville de l'Est, les hommes boivent, les femmes enragent : après la chute du Mur, tout le monde n'a pas pu prendre en marche le train du capitalisme, il roulait trop vite. Se saouler tous les soirs signifie retrouver les forces qui manquent, se sentir important ou grand - et nous en faire rire. Martin, qui avait fui ce monde, comprendra à quel point il lui est cher. L'auteure, jeune Slovaque arrivée en France en 1998, pose un regard plein de tendresse sur les laissés-pourcompte: "Ils détestaient affreusement leur existence, mais pouvaient l'aimer ardemment quand ils étaient ivres." Dans ces villages rudes de l'Est, les gens n'ont jamais su exprimer leurs sentiments, "les mots servaient à travailler, comme les mains", mais Andrea Salajova y parvient dans son 
premier roman, peut-être parce qu'elle l'a écrit en français. "Il ne nous reste qu'à les aimer", dit-elle des héros cabossés de cet "eastern".

\section{Minh Tran Huy}

Voyageur malgré lui

Flammarion

Comment raconter d'où l'on vient, de quelle culture, de quels silences, sans écrire son autobiographie ? Minh Tran Huy a choisi le détour de la fiction. Dans Voyageur malgré lui, Line, la narratrice, prend quelques jours de congé à New York et découvre dans un musée l'existence d'Albert Dadas. Alors qu'elle se passionne pour ce premier cas de fugueur pathologique, elle se souvient d'autres "voyageurs malgré eux" : des membres de sa propre famille surtout, au premier rang desquels son père. Arrivé en France au début des années 1960 pour faire des études, il n'en est jamais reparti. Un père très présent mais d'une grande discrétion sur les drames qu'il a vécus au Viêtnam. Juste avant le naufrage de sa mémoire, d'où seuls émergent encore les souvenirs de son pays natal, il raconte à sa fille sa vie d'avant l'exil. Un livre très délicat sur la transmission.

\section{Valérie Zenatti}

Jacob, Jacob

L'Olivier

Pour Valérie Zenatti, née à Nice, la terre des origines, c'est Constantine de l'Algérie coloniale, quand juifs et Arabes vivaient ensemble, souvent dans la même pauvreté, parlaient la même langue, aimaient les mêmes plats. On suit le court destin de Jacob, jeune juif de Constantine enrôlé en juin 44 pour défendre "une Europe qui avait tué ou laissé mourir ses juifs". Dans une langue charnelle et musicale, Valérie Zenatti décrit, de la
Provence à l'est de la France, la campagne de la $3^{\mathrm{e}}$ division algérienne où combattent côte à côte musulmans, juifs et Français d'Algérie. Comme nombre de ses camarades, Jacob y perdra la vie. Un peu moins de vingt ans plus tard, une autre guerre oblige sa famille à traverser à son tour la Méditerranée. La mort de Cheik Raymond, la star de la musique arabo-andalouse, a sonné le glas de la communauté juive d'Algérie. Dans ce roman nourri de souvenirs familiaux, la fiction et l'émotion ont aussi toute leur place.

\section{Les membres du jury 2015}

- Président du jury, Julien Delmaire, lauréat 2014 pour Georgia, Grasset

- Arlette Farge, historienne

- Michaël Ferrier, écrivain, lauréat 2011 pour Sympathie pour le fantôme (Gallimard)

- Mustapha Harzoune, critique littéraire, Hommes \& Migrations

- Emmanuel Khérad, journaliste culturel, La Librairie francophone, France Inter

- Valérie Marin La Meslée, critique littéraire au Point

- Léonora Miano, écrivain, prix Femina 2013

- Véronique Ovaldé, écrivain

- Isabelle Quentin-Heuzé, Fondation EDF

- Sébastien Wespiser, libraire

- Un groupe d'étudiants de Paris-XIII, une première $L$ du lycée Charlemagne (Paris IV), une seconde du lycée Elisa-Lemonnier (Paris XII), une seconde du lycée Paul-Valéry (Paris XII), une seconde du lycée BlaiseCendrars (Sevran, 93) 